\title{
Isavuconazonium Sulfate
}

\author{
National Cancer Institute
}

\section{Source}

National Cancer Institute. Isavuconazonium Sulfate. NCI Thesaurus. Code C139756.

The sulfate ester form of isavuconazonium, a prodrug of the triazole antifungal agent isavuconazole, with broad-spectrum antifung al activity. Upon administration, isavuconazonium sulfate is hydrolyzed by plasma esterases to yield the active moiety isavuconazole. Isavuconazole binds to and inhibits the fung al cytochrome P450 family enzyme lanosterol 14-alpha-demethylase (CYP51), which catalyzes the demethylation of lanosterol to yield ergosterol, an important component of the fung al cell membrane. Inhibition of CYP51 leads to a decrease in fung al ergosterol production and disrupts synthesis of the fungal cell membrane, which decreases membrane integ rity, increases cell membrane permeability and promotes the loss of essential intracellular elements. This results in fungal cell lysis and death. 